\title{
Magnetite ferrofluids stabilized by sulfonato-calixarenes $\dagger$
}

\author{
Suk Fun Chin, ${ }^{a}$ Mohamed Makha, ${ }^{* a}$ Colin L. Raston ${ }^{* a}$ and Martin Saunders ${ }^{b}$ \\ Received (in CAMBS) 20th December 2006, Accepted 19th January 2007 \\ First published as an Advance Article on the web 16th February 2007 \\ DOI: $10.1039 / \mathrm{b} 618596 \mathrm{~g}$
}

Magnetite $\left(\mathrm{Fe}_{3} \mathrm{O}_{4}\right)$ nanoparticles stabilised by sulfonatocalixarene macrocycles are readily accessible by a rapid in situ co-precipitation, and exhibit ferro-fluidic and superparamagnetic behaviour.

Superparamagnetic magnetite $\left(\mathrm{Fe}_{3} \mathrm{O}_{4}\right)$ nanoparticles have been widely studied for various scientific and technological applications such as magnetic storage media, ${ }^{1}$ contrast agents for magnetic resonance imaging (MRI), ${ }^{2}$ biolabelling and separation of biomolecules, ${ }^{3}$ and magnetic targeted drug delivery. ${ }^{4}$ All these applications require the magnetic nanoparticles to be chemically stable, have particle size $<20 \mathrm{~nm}$ with a narrow size distribution, and to be well dispersed in aqueous medium. ${ }^{5}$ Preparation of stable magnetic nanoparticles is a challenge as the particles have large surface area to volume ratios and thus they tend to aggregate to reduce their surface energy. In addition, there are strong magnetic dipole-dipole attractions between the particles that also cause the particles to aggregate.

Tetramethylammonium hydroxide (TMAOH) is well known as a surfactant to stabilize such magnetite nanoparticles. ${ }^{6}$ However, TMAOH is highly basic and is not biocompatible. Stabilization of magnetic nanoparticles can also be achieved by coating the particle surfaces with organic surfactants or polymers. Oleic acid ${ }^{7}$ and lauric $\operatorname{acid}^{8}$ are commonly used for this purpose. Khalafalla and Reimers ${ }^{9}$ and Wooding et al. ${ }^{10}$ have stabilised suspensions of magnetite using various saturated and unsaturated fatty acids as primary and secondary surfactants. Synthetic polymers and biopolymers have also been used to coat and stabilize magnetic nanoparticles. Examples of the former include poly(vinyl alcohol), ${ }^{11}$ poly(acrylic acid), ${ }^{12}$ and triblock co-polymers (PEOCOOH-PEO). ${ }^{13}$ Among the biopolymers used to stabilize magnetite nanoparticles are dextran ${ }^{14}$ and alginic acid. ${ }^{15}$ Most of this work involves adding the surfactant to the preformed magnetite nanoparticles.

One of the drawbacks of the above approach is that the particle size distribution is difficult to control, and the process is timeconsuming requiring further work up. On the other hand, the synthesis of magnetite nanoparticles in the presence of a surfactant

${ }^{a}$ Centre for Strategic Nano-Fabrication, School of Biomedical, Biomolecular and Chemical Sciences, The University of Western Australia, 35 Stirling Highway, Crawley, WA 6009, Australia. E-mail: clraston@chem.uwa.edu.au; mmakha@chem.uwa.edu.au; Fax: (618) 64881005; Tel: (618) 64881572

${ }^{b}$ Centre for Microscopy Characterisation and Analysis, The University of Western Australia, 35 Stirling Highway, Crawley, WA 6009, Australia

$\dagger$ Electronic supplementary information (ESI) available: synthesis and experimental details TEM, Diffraction patterns, DLS, zeta potential and FTIR for coated magnetite with calix $[n]$ arene sulfonates. See DOI: $10.1039 / \mathrm{b} 618596 \mathrm{~g}$ can circumvent aggregation and control the size of the nanoparticles. Yaacob et al. have prepared magnetite nanoparticles at room temperature $<15 \mathrm{~nm}$ in diameter with a narrow size distribution of the particles which are inside vesicles based on a variety surfactants, namely cetyltrimethylammonium bromide (CTAB) and dedecylbenzenesulfonic acid (DBSA). ${ }^{16}$

$p$-Sulfonato-calix $[n]$ arenes are cyclic phenolic oligomers with a hydrophobic cavity, which can form host-guest inclusion complexes in a similar way to cyclodextrins. Such water soluble calixarenes display interesting biological properties such as antiviral and anti-bacterial activity, ${ }^{17}$ and form inclusion complexes with a variety of small molecules. ${ }^{18}$ Complexes with hydrophobic drugs impart increased solubility of the drug molecules in aqueous medium. ${ }^{19}$ Complexation of Bovine Serum Albumin (BSA), an arginine- and lysine-rich protein, with sulfonato calixarenes has been demonstrated by Memmi et al. ${ }^{20}$ Furthermore, both in vitro and in vivo toxicity studies show that sulfonato-calixarenes have low toxicity. ${ }^{21}$ Overall, $p$-sulfonato-calixarenes have potential for biomedical applications, and in this context we note that sulfonato-calix[4,5,6,8]arenes act as surfactants in stabilizing trans- $\beta$-carotene nanoparticles. ${ }^{22}$

In this study we report the stabilization of superparamagnetic magnetite nanoparticles by coating them with $p$-sulfonato-calix[6 and 8]arenes, $\mathbf{1}, n=6,8$, and sulfonated $p$-benzylcalix[4,5,6 and 8]arenes, 2, $n=4,5,6,8$, Fig. 1.\$ Remarkably, stable ferrofluids are formed by a rapid and simple in situ co-precipitation from a solution of $\mathrm{Fe}$ (II) and $\mathrm{Fe}$ (III) chloride in the appropriate ratio, with aqueous ammonia, in the presence of the $p$-sulfonato-calixarenes and sulfonated $p$-benzylcalixarenes. The calixarenes not only serve as surfactants to stabilize the magnetite nanoparticles, they also functionalize the magnetite nanoparticles for potential biomedical applications, Fig. 1. To the best of our knowledge, this is the first report in the literature of coating magnetite nanoparticles with calix $[n]$ arenes.

The formation of superparamagnetic magnetite nanoparticles was confirmed by SQUID measurements and TEM, while the

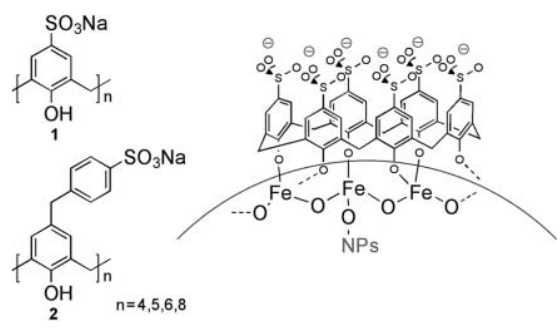

Fig. $1 p$-Sulfonato-calix $[n]$ arenes and sulfonato $p$-benzylcalix $[n]$ arenes showing a possible mode of interaction of $1, n=6$, at the surface of the nanoparticles (NPs). 